\title{
Remodeling of Ventricular Conduction Pathways in Healed Canine Infarct Border Zones
}

Robert A. Luke and Jeffrey E. Saffitz

Cardiovascular Division and Department of Pathology, Washington University School of Medicine, St. Louis, MO

\begin{abstract}
Remodeling of myocyte interconnections may be an important determinant of ventricular tachycardia in regions bordering healed infarcts. We used quantitative electron microscopy to characterize the distribution of gap junctions in $\mathbf{1 0}$ canine left ventricles 3-10 wk after coronary occlusion. In three normal canine left ventricles analyzed ultrastructurally, myocardial gap junctions were distributed anisotropically; gap junction profile length was significantly greater in the transverse than in longitudinal planes of section. In infarct border zone tissues, the normal anisotropic distribution was completely abolished and fewer gap junctions per unit intercalated disk length were observed. Analysis of individual gap junction profile length distributions revealed selective disruption of the largest gap junctions that collectively comprised only $9.6 \%$ of total junction profiles, but encompassed nearly $\mathbf{4 0} \%$ of aggregate gap junction length in the transverse plane of section. Three-dimensional reconstructions of myocyte interconnections by high resolution quantitative light microscopy of serial sections demonstrated a reduction in the number of cells connected by intercalated disks to a single myocyte from 11.2 \pm 1.0 in normal myocardium to $6.5 \pm 1.3$ in border zone tissues $(P<0.001)$. Connections of cells in primarily side-to-side apposition were reduced by $\mathbf{7 5 \%}$, whereas primarily end-to-end connections were reduced by only $22 \%(P<0.05)$. These alterations would disproportionately enhance axial resistivity in the transverse direction, potentially contributing to development of reentrant arrhythmias. (J. Clin. Invest. 1991. 87:1594-1602.) Key words: Ventricular tachycardia • gap junctions • electron microscopy • fibrosis • intercalated disks
\end{abstract}

\section{Introduction}

The pathogenesis of reentrant arrhythmias requires both unidirectional block and slow conduction within the reentrant circuit (1). While a considerable body of evidence has implicated alterations of active membrane properties in the development of conduction abnormalities, more recent observations have indicated that changes in passive myocardial resistivity likely play a role as well (2-8). Slow, heterogeneous electrical propaga-

Address correspondence to Dr. Jeffrey E. Saffitz, Department of Pathology, Box 8118, Washington University School of Medicine, 660 So. Euclid Ave., St. Louis, MO 63310.

Received for publication $8 \mathrm{May} 1990$ and in revised form $28 \mathrm{No}$ vember 1990.

J. Clin. Invest.

(c) The American Society for Clinical Investigation, Inc. 0021-9738/91/05/1594/09 \$2.00

Volume 87, May 1991, 1594-1602 tion and complex fractionated electrograms have been observed in fibrotic myocardium with normal cellular active membrane properties, which has led to speculation that alterations of specific patterns of myocyte interconnections in regions bordering healed infarcts may be an important component of the anatomic substrate of reentrant ventricular tachycardia $(3,5,6,8-14)$.

Spach and Dolber (15) have demonstrated loss of electrical coupling of atrial myocytes oriented in side-to-side apposition due to progressive interstitial fibrosis associated with aging. Others, such as Dillon et al. (5), have characterized reentrant circuits in healed infarct border zones and have suggested that alterations in side-to-side connections similar to those described in the aged atria may be important determinants of arrhythmogenesis. The present study is an extension of the work of Spach and Dolber and was designed to rigorously quantify the three-dimensional distribution of myocyte interconnections in selected regions of the healed infarct border zones known to be sites of slow heterogeneous propagation critical in reentrant arrhythmogenesis.

We have recently characterized the three-dimensional structure and distribution of gap junctions and patterns of cellular interconnection in normal canine left ventricular myocardium using light and electron microscopic morphometry $(16,17)$. We observed an extraordinary level of structural complexity. For example, the typical canine ventricular myocyte has on its surface $\sim 100$ gap junctions located in specific domains of the intercalated disk and having specific sizes, shapes, and orientations (16). A subset of these junctions includes exceptionally long (up to $8 \mu \mathrm{m}$ in length), ribbon-shaped gap junctions oriented with their long axes transverse to the myocyte longitudinal axis, an arrangement that is likely to minimize shear forces on stiff patches of densely packed channels connecting adjacent cells. These junctions are located in interplicate regions of the intercalated disk. The remaining gap junctions are small discoid structures located within the plicate segment of the intercalated disk. Representative electron micrographs of long interplicate and smaller plicate segment gap junctions are shown in Fig. 1.

We have also shown that an individual cell is connected by intercalated disks to $\sim 10$ other cells in variable degrees of side-to-side and end-to-end packing (16). In the present study, we applied a similar quantitative structural approach to characterize the effects of interstitial fibrosis on intercellular junctions in epicardial regions bordering healed canine infarcts. The results indicate that accumulation of interstitial collagen coincides with the disruption and remodeling of intercellular junctions. Long transversely oriented gap junctions appeared to be especially vulnerable and were essentially absent from infarct border zone myocardium. These structural derangements would enhance the anisotropy of cellular connections and disproportionately increase the axial resistivity encountered by a wavefront propagated transverse to the long fiber axis. 


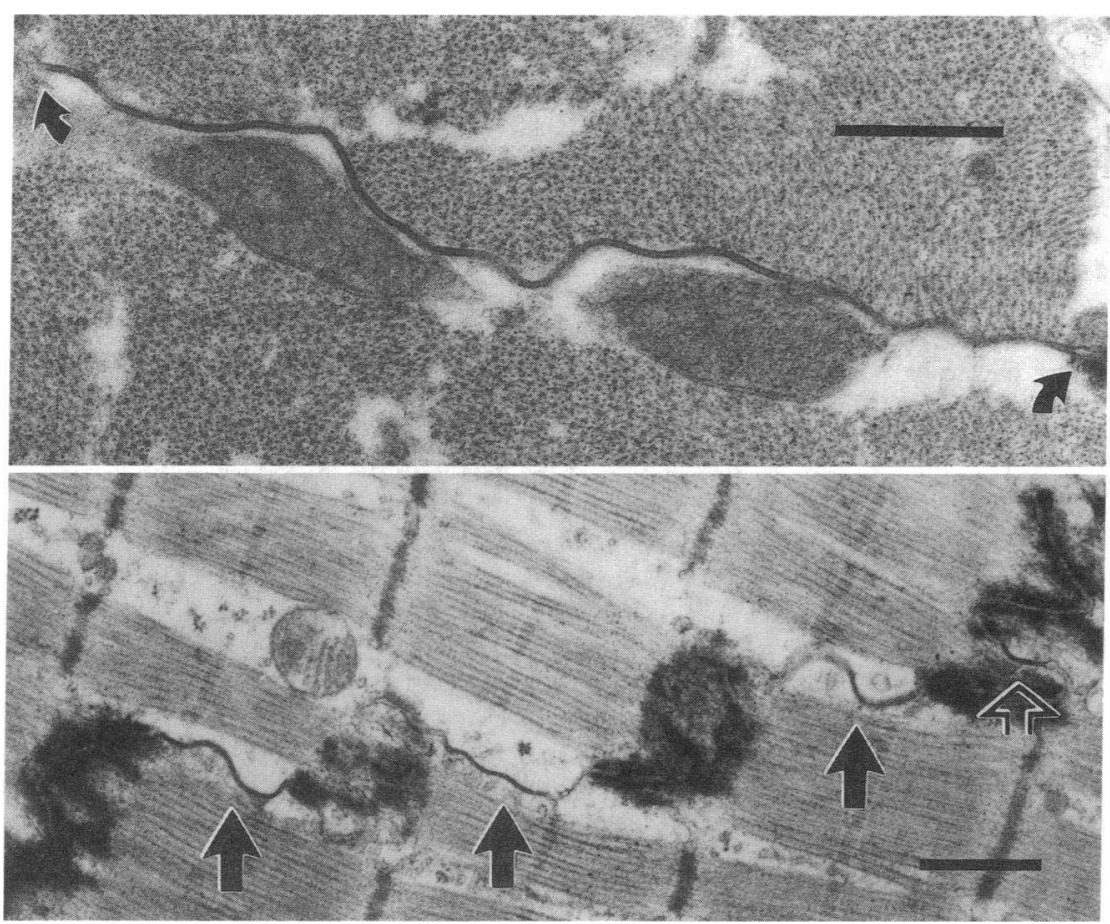

Figure 1. Electron micrographs of gap junction profiles in normal canine epicardial tissue. A long, ribbon-shaped gap junction (between arrows) is readily apparent in the transverse plane of section (top panel). Small discoid gap junctions in plicate segments (open arrow) and short axis views of the long, ribbon-like junctions in interplicate segments (closed arrows) are shown in the longitudinal plane of section (bottom panel). Bar, $1.0 \mu \mathrm{m}$ in each panel.

\section{Methods}

Tissue preparation for electron microscopic morphometry. Tissue was obtained from 10 adult mongrel dogs of either sex that had previously been subjected to coronary occlusion and from 3 adult mongrel control dogs. Surgical ligation of the left anterior descending coronary artery was performed as previously described (18). After convalescence under approved veterinary care for 3-10 wk, the animals were reanesthetized with sodium thiopental $(20 \mathrm{mg} / \mathrm{kg}$ i.v. $)$ and the hearts were excised as previously described (17). Tissue samples $\sim 1 \times 1 \times 0.2 \mathrm{~cm}$ in size were dissected from left ventricular epicardial regions overlying transmural infarcts in the experimental animals and from corresponding left ventricular regions of control animals. The tissue was immersed in modified Karnovsky's fixative ( $2.0 \%$ glutaraldehyde and $1.0 \%$ paraformaldehyde in $0.08 \mathrm{M}$ sodium cacodylate buffer containing $2 \mathrm{mM}$ calcium chloride, $\mathrm{pH} 7.4$ ) and fixed at $4^{\circ} \mathrm{C}$ for at least $12 \mathrm{~h}$.

10-20 tissue blocks measuring $1 \times 1 \times 0.5 \mathrm{~mm}$ were cut from each epicardial tissue sample. A dissecting microscope was used to determine fiber orientation and to aid in selection of blocks composed of an interspersion of myocytes and collagen. Individual blocks were processed for electron microscopy by postfixation for $15 \mathrm{~min}$ in $1.0 \%$ osmium tetroxide, dehydration in ethanol, and embedment in Spurr's resin. Thick sections $(0.5 \mu \mathrm{m})$ of each block were examined light microscopically to verify that myocytes had been cut in planes either parallel or perpendicular to the longitudinal fiber axis and to determine the structural composition of the sample.

A spectrum of structural features ranging from $100 \%$ collagenous scar to bundles of normal appearing myocytes separated from other bundles by collagenous septa or even entirely normal myocardium was observed in $\sim 150$ blocks examined from epicardial infarct border zones. In view of the wide variability in the structural composition of border zone regions and the expectation that the corresponding effects on intercellular connections would range from total disruption in fully scarred regions to negligible alterations within normal appearing myocardium, these regions were not sampled randomly. Rather, we selected specific samples for analysis that were composed of cardiac myocytes separated by diffuse interstitial fibrosis with maintenance of normal fiber anisotropic orientation. Fibrosis in these samples was exclusively interstitial and did not appear to have been formed by replacement of necrotic myocytes.

Because gap junction membranes are oriented anisotropically in normal canine ventricular myocardium, intercellular junctions had to be analyzed morphometrically in three planes of section orthogonal to the longitudinal fiber axis. In normal tissue, this was accomplished by sectioning independent tissue samples in each orthogonal plane, as reported previously (16). In the infarct border zone, however, structural heterogeneity made this approach impractical. Thus, sections of individual border zone tissue blocks were analyzed morphometrically in three planes orthogonal to the long fiber axis by first cutting sections in one longitudinal plane (e.g., parallel to the long fiber axis and parallel to the epicardial surface), then reorienting the block in the microtome by $90^{\circ}$ to permit sectioning in the other longitudinal plane (parallel to the long fiber axis and perpendicular to the epicardial surface), and finally reorienting the block a second time to prepare sections transverse to the long fiber axis.

A total of 10 blocks of border zone tissue ( 1 from each of 10 animals subjected to coronary occlusion) and a total of 5 blocks from 3 control animals ( 2 blocks from each of 2 control animals and 1 block from 1 control animal) were analyzed. Ultrathin sections were prepared from each orientation and collected on 200-mesh copper-rubidium grids after fiber orientation had been verified with light microscopy of 0.5 $\mu \mathrm{m}$ thick sections. Ultrathin sections were stained with uranyl acetate and lead citrate, examined with an electron microscope (model EM200; Philips Electronic Instruments, Mahweh, NJ), and photographed with Eastman 5302 fine grain release positive $35-\mathrm{mm}$ film (Eastman Kodak Co., Rochester, NY).

Quantitative morphometric analysis of electron micrographs. At least five test regions were randomly selected for ultrastructural analysis from each group of ultrathin sections cut in each orientation of each tissue block as previously described (16). Individual test regions were photographed at a final print magnification of $\times 5,000$. Then, all portions of each test area containing intercalated disks and gap junction profiles were rephotographed for further analysis at a final print magnification of $\times 24,000$. A total of 432 high power micrographs of control tissue and 875 micrographs of fibrotic tissue were analyzed.

Intercalated disk and gap junction profile lengths were measured in 
each high power micrograph using an electronic stylus and digitizing tablet (Houston Systems Inc., Austin, TX). Data were expressed as gap junction profile length per $100 \mu \mathrm{m}$ intercalated disk length, or gap junction profile length per unit myocyte area. The percentages of total section area occupied by cardiac myocytes and by interstitial fibrosis were measured in $\times 5,000$ micrographs of each individual test area by placing a point array $(1.0 \mathrm{~cm}$ interpoint distance $)$ over each $8 \times 10 \mathrm{inch}$ micrograph and counting the number of points located over interstitial and noninterstitial structures (19).

In addition to measuring gap junction profile lengths, individual gap junctions were categorized as being located in either plicate or interplicate regions of the intercalated disk. Plicate region gap junctions were defined as those located within the interdigitating adhesive processes of the disk, while interplicate junctions were located in junctional membranes between plicate segments.

Light microscopic analysis of patterns of intercellular connection. Additional tissue blocks were analyzed with light microscopy to determine the number of cardiac myocytes to which an individual myocyte of normal and border zone tissue was connected by intercalated disks and to characterize the relative side-to-side or end-to-end orientation of these interconnections. Blocks measuring $\sim 1 \times 1 \times 0.5 \mathrm{~mm}$ were dissected from epicardial regions overlying healed transmural infarcts and from corresponding regions of control tissue, postfixed for $15 \mathrm{~min}$ in $1.0 \%$ osmium tetroxide containing $1.0 \%$ potassium ferrocyanide, dehydrated in ethanol, and embedded in Spurr's resin. The addition of potassium ferrocyanide enhanced sarcolemmal staining, thereby facilitating identification of cell borders and specifically junctional membrane at the light microscopic level of resolution (16). A cellular interconnection was tabulated only after unequivocal identification of an intercalated disk between the cells. It was assumed that the presence of one or more intercalated disks connecting two cells at the light microscopic level of resolution indicated electrical interconnection at gap junctions.

A series of 40 consecutive sections, each $\sim 1.5 \mu \mathrm{m}$ in thickness, was cut in a plane longitudinal to the long fiber axis from each of four blocks of border zone tissue and four blocks of normal myocardium. Sections were stained with toluidine blue and each section was photographed at a final print magnification of $\sim 1,000$. Five individual index cardiac myocyte profiles were randomly selected from the 20th section of each set of 40 serial sections, and these index cells and all neighboring myocytes to which they were connected by intercalated disks were delineated and counted by identifying, in their entirety, profiles of these cells in serial sections. In addition to determining the number of cells connected to the five index cells in each set of serial

I

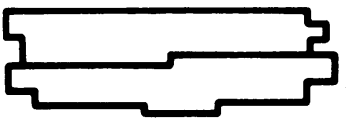

II

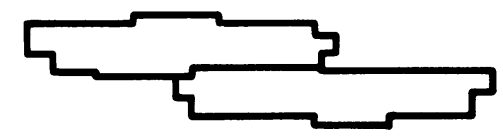

III

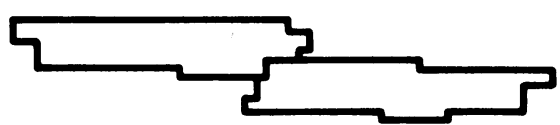

立

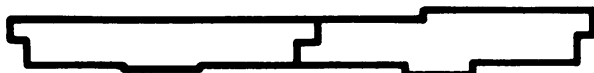

Figure 2. Diagram of types of intercellular connections used in threedimensional reconstructions. sections, the spatial distribution of each interconnection was categorized on the basis of its relative side-to-side or end-to-end orientation according to the following system: Type I, > 75\% of the lateral borders of the two cells overlapped; Type II, 25-75\% lateral border overlap; Type III, $<25 \%$ lateral border overlap and $<50 \%$ end-to-end overlap Type IV, $<25 \%$ lateral border overlap and $>50 \%$ end-to-end overlap. Schematic diagrams of these types of connections are illustrated in Fig. 2.

Statistical analysis. Data were expressed as means \pm SD. Morphometric data were analyzed by nonparametric Wilcoxon tests (20) except as noted. Histograms of gap junction profile lengths were examined by analysis of covariance (20). Because of the skewed lognormal distributions, analysis of covariance was performed following logarithmic transformation of the data with the log values as the dependent variables. Independent variables included the $\log$ of the bin, the group, and the interaction between the two. The first two bins in each histogram were eliminated as outliers that violated the overall pattern of the distribution. Accordingly, the statistical analysis does not apply to the two smallest bins of gap junction profile length. The data were also tested with $\chi^{2}$ analysis of the percentage of profiles above and below an arbitrary cut off of $3.0 \mu \mathrm{m} . \chi^{2}$ analysis was also used to test the dichotomous distribution of gap junctions in plicate and interplicate regions of intercalated disks (20). Nonparametric Wilcoxon tests were used to test differences in each connection type between normal regions and border zone regions (shown in Table I). The significance of the percent reductions of specific intercellular connection types was tested using analysis of covariance on a rank transformation of the data.

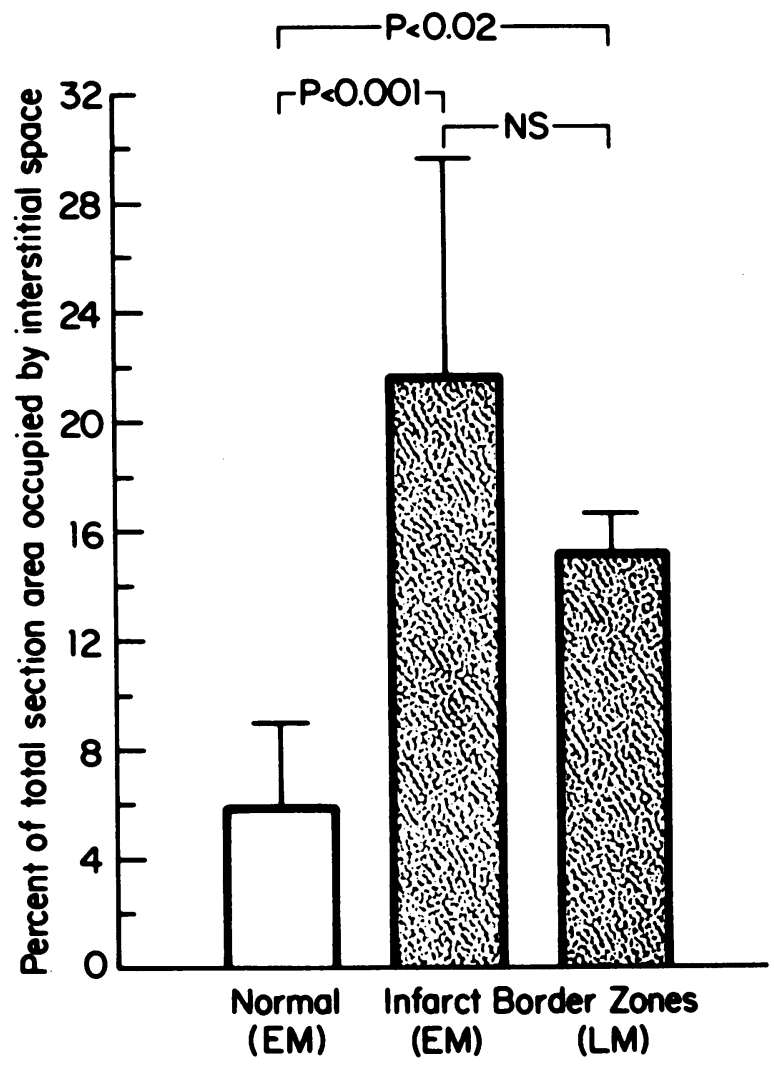

Figure 3. Relative proportions of test areas occupied by interstitial space (all nonmyocyte structures) in normal $(n=15)$ and border zone regions $(n=30)$ analyzed with electron microscopy (EM) and in border zone tissues $(n=4)$ analyzed with light microscopy (LM) of serial sections. Interstitial space in normal samples analyzed with light microscopy was $5.4 \pm 0.6 \%$ of total section area $(P=$ NS compared with normal samples analyzed ultrastructurally). 


\section{Results}

Electron microscopic morphometry of intercellular connections. The relative amounts of total section area occupied by interstitial space in control and infarct border zone test regions are shown in Fig. 3. In normal tissues, the interstitium occupied $\sim 6 \%$ of section area, a value similar to those reported in previous morphometric studies of canine ventricular myocardium (21). In border zone regions, interstitial space encompassed $\sim 22 \%$ of total sectional area in samples analyzed by electron microscopy and $\sim 15 \%$ in samples analyzed by light microscopy. Expansion of the interstitial space was due to accumulation of bundles of collagen fibrils, as shown in a representative electron micrograph (Fig. 4).

Results of morphometric analysis of gap junction size and distribution in normal and infarct border zone regions are shown in Figs. 5 and 6. Because no significant differences were observed in any parameter analyzed in the longitudinal planes perpendicular and parallel to the epicardial surface, data from these two planes were pooled and are referred to in Figs. 5-7 as the longitudinal planes of section. As shown in Fig. 5, the anisotropic distribution of gap junction profile length is readily apparent in normal canine myocardium. Whether expressed as gap junction profile length per unit myocyte area or per unit intercalated disk length, values observed in the transverse planes of section were significantly greater than those in the longitudinal planes. As demonstrated previously, the anisotropic distribution is a consequence of long, ribbon-shaped gap junctions, located in interplicate segments, and having their long axes oriented transverse to the long fiber axis. Thus, as shown in Fig. 6, the average individual gap junction profile length in normal ventricular myocardium was greater in the transverse than in the longitudinal planes of section (1.35 vs. $0.87 \mu \mathrm{m}$, respectively). However, the number of gap junction profiles was the same in both orientations (12.9 vs. 13.2 profiles $/ 100 \mu \mathrm{m}$ intercalated disk length in longitudinal and transverse planes of section, respectively [Fig. 6]).

In marked contrast to the pattern observed in normal myocardium, the anisotropic distribution of gap junction length was significantly altered in the fibrotic myocardium of the in-

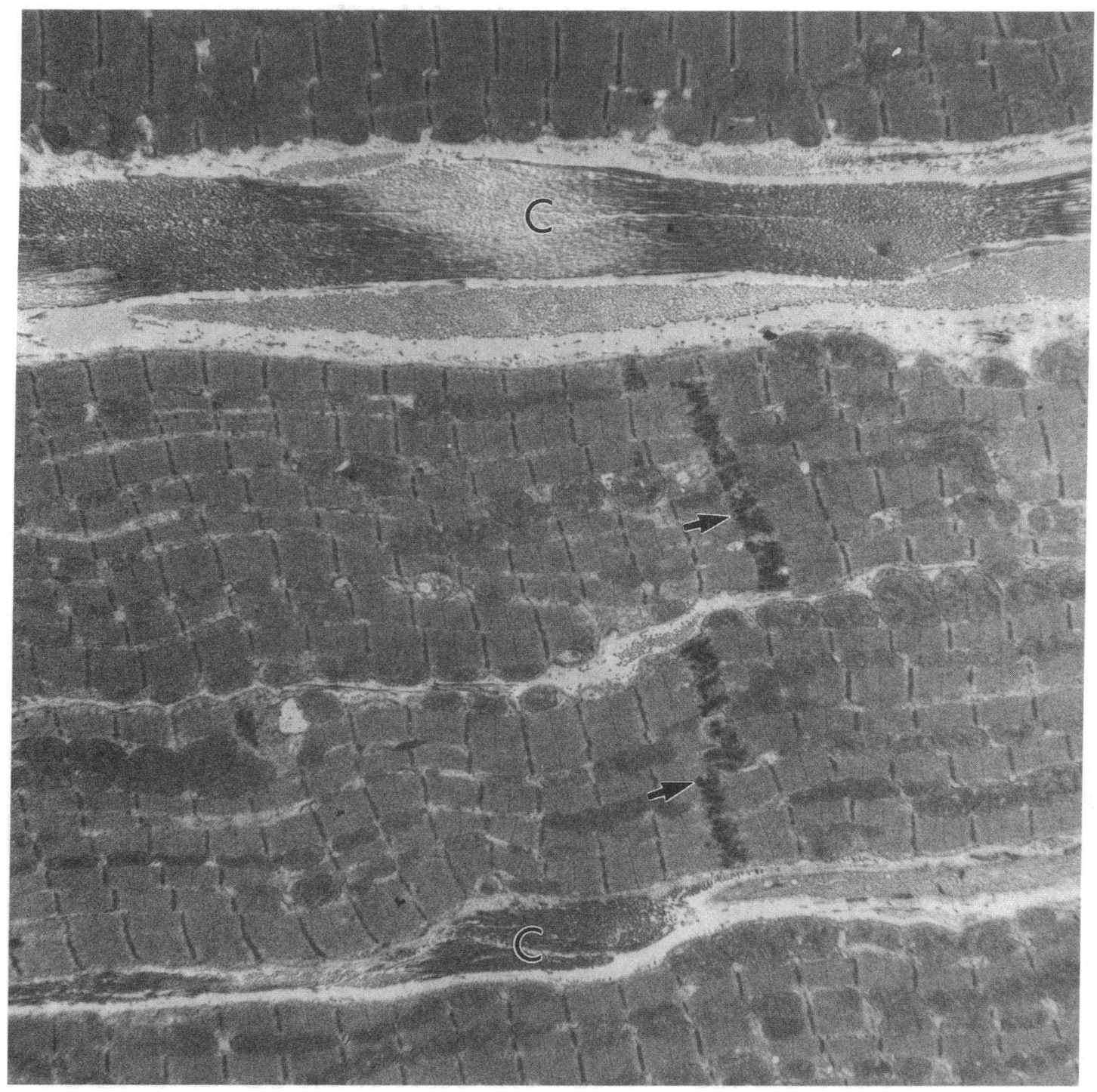

Figure 4. Electron micrograph of a typical epicardial border zone region cut in a longitudinal plane of section. Interstitial bundles of collagen $(C)$ separating cells are readily apparent. Intercalated disks connecting cells in end-to-end fashion are identified by arrows. $\times 5000$. 
farct border zone (Fig. 5). Compared with normal tissue, there was a significant reduction in gap junction profile length in both longitudinal and transverse planes of section, but this decrement was far greater in the transverse plane, such that gap junction length per unit myocyte area or intercalated disk length became equivalent in both orientations. The reduction in aggregate gap junction profile length in border zone tissues was the result of there being fewer individual profiles per unit disk length in both longitudinal and transverse orientations (Fig. 6), although the difference in the transverse plane failed to achieve statistical significance due to a relatively large standard deviation. Nevertheless, these data indicate that the increase of interstitial fibrosis coincided with a disruption of cell junctions with fewer individual gap junction profiles per unit intercalated disk length. Moreover, the average length of an individual gap junction in border zone samples was significantly reduced in the transverse plane but not in the longitudinal planes (Fig. 6). These observations suggest that long, ribbon-shaped gap junction profiles, known to have their long dimensions oriented transverse to the longitudinal fiber axis, may be selectively disrupted in fibrotic tissue.

Histograms of the distribution of individual gap junction profile lengths in the transverse and longitudinal planes of section of normal and infarct border zone samples are shown in Fig. 7. The greater aggregate and mean individual gap junc-
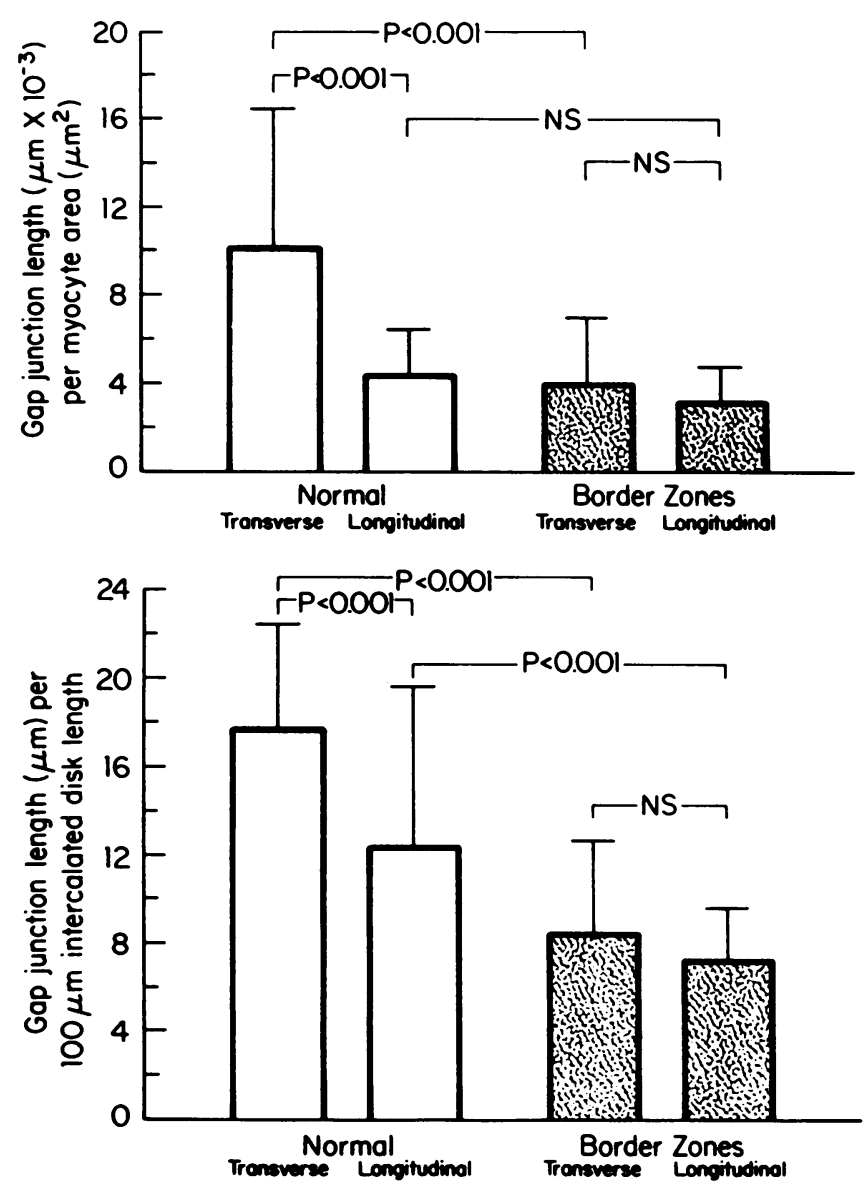

Figure 5. Total gap junction profile length per $\mu \mathrm{m}^{2}$ myocyte area (top panel) and per $100 \mu \mathrm{m}$ intercalated disk length (bottom panel) measured in transverse and longitudinal planes of section in normal and border zone test areas.
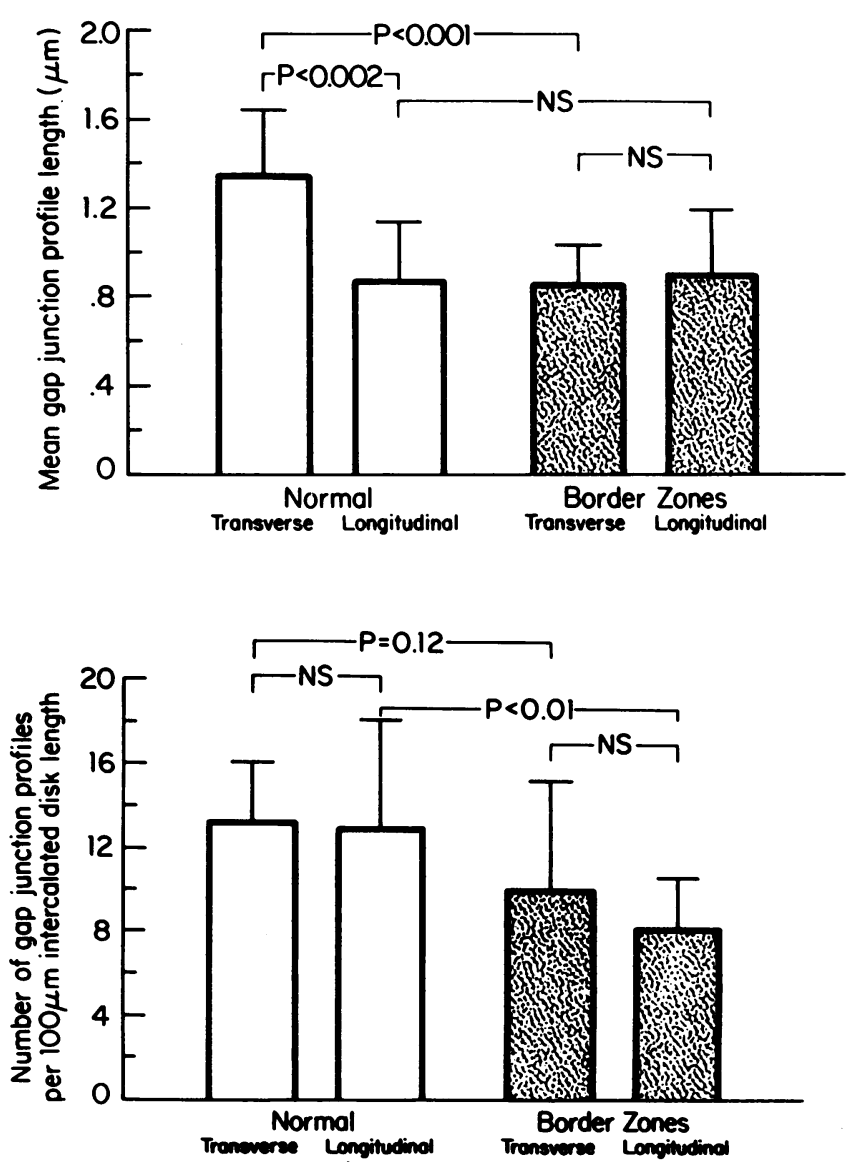

Figure 6. Mean gap junction profile length $(\mu \mathrm{m})($ top panel $)$, and the number of individual gap junction profiles per $100 \mu \mathrm{m}$ intercalated disk length (bottom panel) in transverse and longitudinal planes of section in normal and border zone test areas.

tional profile lengths observed in the transverse compared with the longitudinal planes in normal tissue (demonstrated in Figs. 5 and 6) are seen in Fig. 7 to be due to a subset of long gap junctions (arbitrarily defined as having a profile length $>3.0$ $\mu \mathrm{m}$ ), apparent only in the transverse plane of section. In the "normal transverse" histogram, 17 of 178 gap junction profiles were $>3.0 \mu \mathrm{m}$ in length. Although only $9.6 \%$ of the total number of profiles, these junctions comprised $37.5 \%$ of the aggregate gap junctional length in the transverse plane of section. Because the "short" axes of these long, ribbon-shaped gap junctions are oriented parallel to the longitudinal fiber axis, few long profiles are observed in normal tissue cut in the longitudinal planes of section (Fig. 1).

In contrast to the transverse profile length distribution of normal tissue, infarct border zone samples showed a marked paucity of long gap junction profiles in the transverse plane (Fig. 7, bottom left). Only 2 of $244(<1 \%)$ profiles in the "border zone transverse" histogram had a length $>3.0 \mu \mathrm{m}$, compared with $9.6 \%$ in normal tissues $(P<0.001)$. Thus, loss of the anisotropic junction distribution and reduction of the average gap junction profile length in the transverse plane of infarct border zone samples (shown in Figs. 5 and 6) were due to selective disruption of the longest gap junction profiles.

In the longitudinal planes of section, no significant differences were found in the gap junction length distribution of 

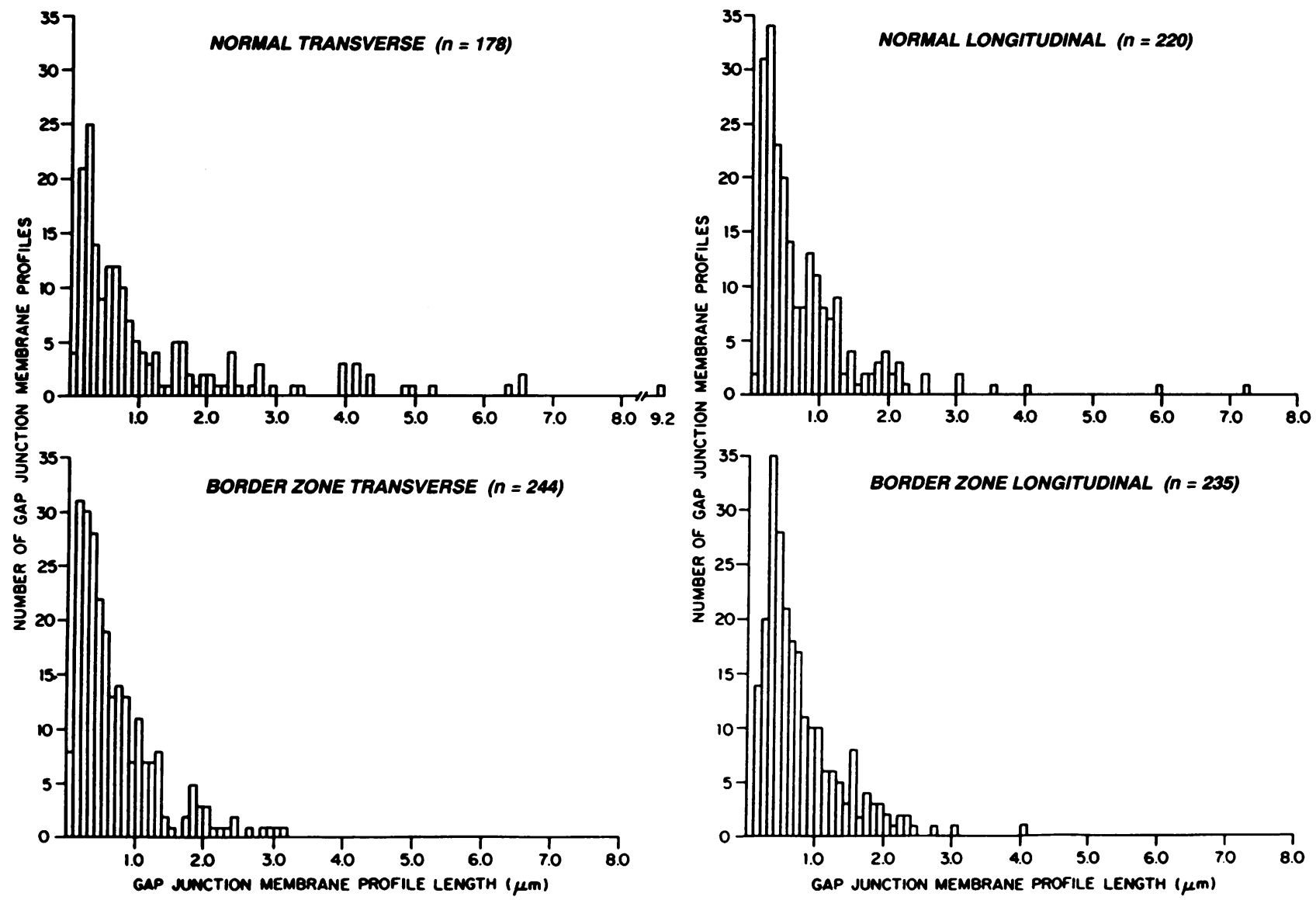

Figure 7. Histograms of gap junction profile length distributions in transverse and longitudinal planes of section in normal and border zone test areas. Using analysis of covariance, significant differences were found between normal longitudinal and normal transverse planes $(P=0.032)$, reflecting the normal anisotropic distribution of gap junctions, and between normal transverse and border zone transverse planes $(P<0.0001)$, indicating disruption and loss of long, ribbon-shaped gap junctions. No significant differences were observed between normal longitudinal and border zone longitudinal, or between border zone transverse and border zone longitudinal.

normal and border zone tissues (Fig. 7), consistent with the observation that the average individual gap junction profile length seen in the longitudinal planes did not change in fibrotic tissue (Fig. 6). Because the short axes (longitudinal dimensions) of the long, transversely oriented, ribbon-shaped junctions have been shown previously to have a mean length of $\sim 1 \mu \mathrm{m}$, selective disruption of these junctions in fibrotic tissue would not result in a decrease in the average profile length observed in longitudinal planes, but would cause reduction in aggregate profile length and the total number of gap junction profiles per unit intercalated disk, as shown in Figs. 5 and 6.

The long, ribbon-shaped gap junctions of normal ventricular myocardium have been shown previously to reside exclusively in interplicate segments, whereas smaller discoid shaped junctions are located within interdigitating adhesive elements of the plicate segment of the intercalated disk (16). Selective disruption of these long, interplicate segment gap junctions in infarct border zone tissue would be expected to decrease the proportion of total gap junctions residing in interplicate segments. Accordingly, the percentages of total gap junction profiles located in interplicate segments of normal tissues and infarct border zone samples were assessed in both longitudinal and transverse planes of section. In normal tissues, $66 \%$ of all gap junction profiles were found in interplicate segments, while the remaining gap junction profiles were located within plicate segments. In fibrotic samples, the proportion of total gap junction profiles identified in interplicate segments decreased significantly to $52 \%(P<0.001)$. These data are concordant with observations shown in Fig. 6 that long transversely oriented gap junction are selectively lost in infarct border zone samples.

Light microscopic morphometry of intercellular connections. Whereas results of electron microscopic morphometry showed disruption of intercellular junctions in infarct border zone tissues, these observations did not indicate whether fewer cells were actually interconnected to one another or whether fewer connections existed between any given cell pair without a reduction in the total number of interconnected cells. In addition, because the ultrastructural analysis did not indicate the relative extent to which side-to-side and end-to-end cell interconnections were disrupted in infarct border zones, light microscopic morphometry was performed to answer these questions. Fig. 8 shows representative fields of normal and border zone myocardium stained to enhance cell borders and permit threedimensional reconstruction of cellular interconnections in serial sections. Cellular interconnections were scored only when a clearly identifiable intercalated disk was observed between the cells.

The number of cells connected to an individual ventricular myocyte was reduced significantly from $11.2 \pm 1.0$ in control tissue to $6.5 \pm 1.3$ in infarct border zone samples $(P<0.001)$. 

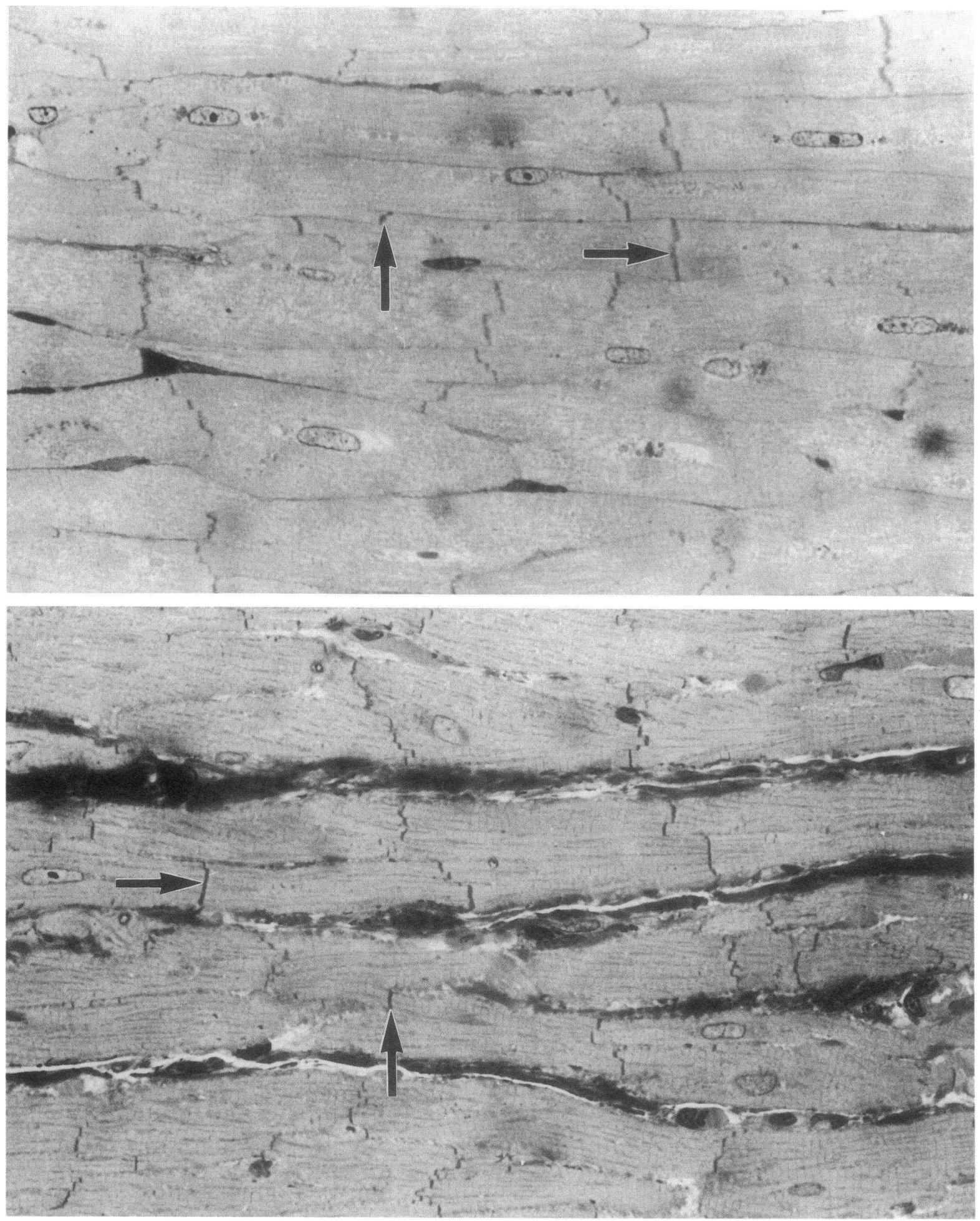

Figure 8. Light micrographs of representative serial sections of normal (top panel) and border zone (bottom panel) test regions showing enhanced sarcolemma staining to facilitate three-dimensional reconstruction of patterns of cell apposition. Examples of intercalated disks connecting cells in primarily side-to-side (vertical arrows) or end-to-end (horizontal arrows) apposition are indicated. An intercellular connection was scored only when a clearly identifiable disk was observed between two cells. $\times 640$.

The value obtained for normal myocardium is in agreement with reported values $(16,22)$. Previously, we observed $9.1 \pm 2.2$ connections per normal canine ventricular myocyte (16), a value that is significantly different $(P<0.01)$ than the value observed in border zone samples in the present study. The loss of cellular interconnections in fibrotic tissue did not occur in a random spatial orientation, but was most marked in cells having primarily side-to-side apposition and least apparent in cells 
connected primarily end-to-end. As shown in Table I, Type I connections (cells having $>75 \%$ lateral border overlap) were diminished by $75 \%$, while Type IV connections ( $>50 \%$ end-toend overlap) were reduced by only $22 \%(P<0.05)$. Intermediate types of connections (Types II and III) were diminished by $35-45 \%$. Thus, connections between cells oriented side-toside were more severely disrupted than connections in cells joined end-to-end.

\section{Discussion}

Results of this quantitative structural study indicate that modest accumulation of collagen in the healed canine infarct border zone that is sufficient to increase the interstitial space from a normal value of $\sim 6 \%$ to $15-20 \%$ while maintaining myocyte fiber anisotropy, is associated with disruption of intercellular junctions and a marked reduction in the number of intercellular connections. Electron microscopic morphometry revealed that the largest connexon arrays in the heart, arranged in long, ribbon-shaped transversely oriented gap junctions, are selectively disrupted. Light microscopic reconstruction of cellpacking patterns indicated that the total number of cell-to-cell contacts is substantially reduced and that side-to-side connections are selectively affected. These structural alterations are summarized in Fig. 9.

Structural abnormalities in regions bordering healed infarcts in the present study varied from extremes of dense collagenous scar to relatively normal myocardium. Often, this range of structural alteration occurred over a distance of only 1-2 $\mathrm{mm}$. Relatively small areas of the border zone were actually composed of the interspersion of myocytes and interstitial collagen that constituted the tissue sample analyzed in the present study. This pattern of interstitial fibrosis with maintained tissue anisotropy is one of multiple potential anatomic substrates of ventricular tachycardia that have been identified in areas bordering both human and canine infarcts $(5,6,8,10-14$, 23-25).

Although the results of the present study indicate that border zone myocytes contain fewer gap junction profiles per unit intercalated disk length and that the longest profiles $(\geq 3.0$ $\mu \mathrm{m})$ are selectively disrupted, it is not known whether only the longest profiles are lost, or whether both long and short profiles become disrupted, or whether long profiles become converted to shorter profiles. Our observations suggest, however, that gap junctional membrane is reduced by a greater extent than overall intercalated disk junctional membrane. Because the longest,

Table I. Distribution of Myocyte Connection Types in Normal and Border Zone Areas

\begin{tabular}{cccc}
\hline $\begin{array}{c}\text { Connection } \\
\text { type }\end{array}$ & $\begin{array}{c}\text { Normal } \\
\text { regions }\end{array}$ & $\begin{array}{c}\text { Border } \\
\text { zone }\end{array}$ & $\begin{array}{c}\text { Percent } \\
\text { reduction }\end{array}$ \\
\hline I & $2.4 \pm 0.8$ & $0.6 \pm 0.6^{*}$ & $75.0^{\ddagger}$ \\
II & $3.1 \pm 1.2$ & $1.9 \pm 1.4^{\S}$ & 37.7 \\
III & $2.2 \pm 0.8$ & $1.2 \pm 0.8^{\S}$ & 45.0 \\
IV & $3.6 \pm 0.9$ & $2.8 \pm 0.8^{\text {II }}$ & 22.0 \\
\hline
\end{tabular}

Values indicate the number of myocytes connected to a single myocyte by each connection type.

$* P<0.001$.

${ }^{\ddagger} P<0.05$ vs. percent reduction of Type IV connections.

$P<0.005$.

" $P<0.05$ vs. normal regions.

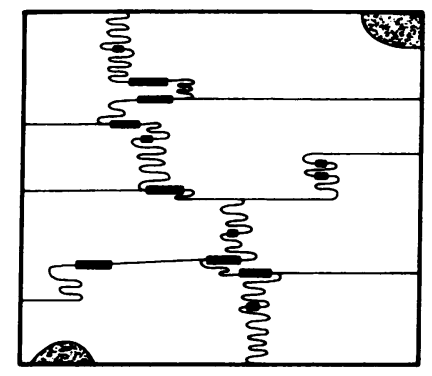

NORMAL LONGITUDINAL

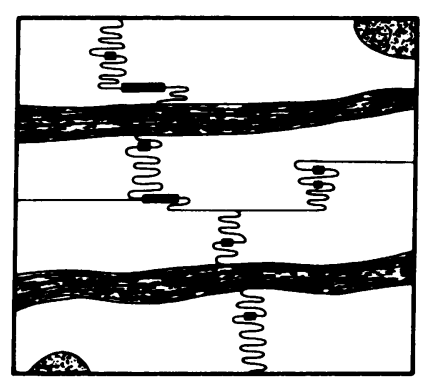

BORDER ZONE LONGITUDINAL

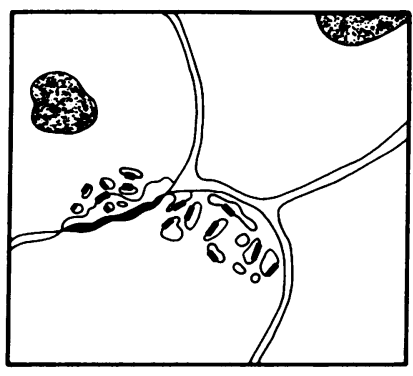

NORMAL TRANSVERSE

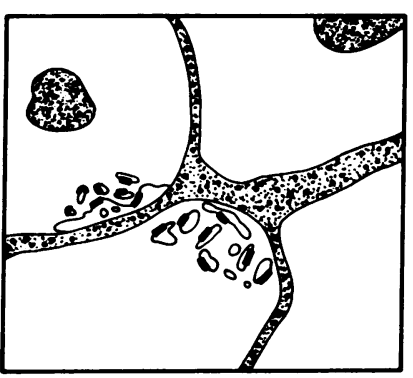

BORDER ZONE TRANSVERSE
Figure 9. Diagram of the effects of interstitial collagen deposition on gap junction distribution and patterns of intercellular connection. Gap junctions are indicated by thickened segments of the junctional membrane connecting cells. A significant reduction in total gap junction membrane length occurs with selective disruption of long, ribbon-shaped interplicate gap junctions. Because the long gap junctions are seen in the short axis in longitudinal planes of section, their loss is observed as a modest reduction in gap junction profile number without a significant decrease in average profile length. In the transverse plane of section, however, their loss results in significant reduction in mean gap junction profile length, producing a more uniform distribution of short gap junction profiles. Reduced coupling is observed at the cellular level to occur disproportionately in cells connected in side-to-side orientation. End-to-end myocyte connections are relatively spared.

transversely oriented gap junctions are often located on the surfaces of myocytes at the edges of intercalated disks (i.e., they are contiguous with nonjunctional sarcolemma) (16), they would likely be more susceptible than shorter profiles to mechanical forces associated with accumulation of interstitial collagen. Plicate segment gap junctions, embedded entirely within the adhesive components of intercalated disks and concentrated at the ends of myocytes, would likely be more resistant to such forces. This interpretation of the observations in the present study assumes that disruption of gap junctions and remodeling of patterns of intercellular connections are caused by mechanical forces attributable to accumulation and organization of collagen bundles oriented parallel to the long myocyte axis. However, such a causal relationship has not been established.

The significant reduction of gap junction surface density delineated with ultrastructural morphometry in border zone tissues indicates that these myocytes are relatively uncoupled and that the tissue would have increased passive internal resistance in comparison with normal myocardium. This observation is consistent with the reduced space constants measured in infarct border zones by Spear et al. (2). Selective disruption of side-to-side intercellular connections demonstrated in three-dimensional reconstructions would appear to enhance the anisotropy of intercellular connections and disproportionately increase resistance to propagation in a direction transverse to the 
long myocyte axis. Whereas any single gap junction may subserve current transfer in either transverse or longitudinal direction, and loss of gap junctions would be expected to increase internal resistance in both directions, disruption of side-to-side connections with relative sparing of end-to-end connections would increase the path length and number of intercellular junctions traversed by a wavefront moving in the transverse direction. These predicted electrophysiologic effects are consistent with the observations of Dillon et al. (5) and Zuanetti et al. (18), who analyzed patterns of propagation in the canine infarct border zone.

Results of the present study also provide insights into mechanisms of fractionated electrograms that have been observed in tissues prone to arrhythmias (26-28). In experimental canine infarcts, complex fractionated electrograms are not apparent during the early phase of infarct healing but occur and persist as the necrotic myocardium is resorbed and replaced by collagenous scar tissue that also affects the spatial orientation and packing of surviving myocytes at the infarct edge $(8,11)$. Gardner et al. have suggested that alterations in intercellular junctions accompanying interstitial fibrosis were responsible for the appearance of fractionated electrograms, and for slow heterogenous propagation in epicardial infarct border regions (11). Before the present study, however, no quantitative structural data on myocyte connections in infarct border zones were available to substantiate this conclusion. Our results provide a more detailed understanding of the structural basis underlying slow propagation, conduction block, and fractionated electrograms, all of which appear to be important determinants of arrhythmogenesis.

\section{Acknowledgments}

We gratefully acknowledge the assistance of Dr. Giulio Zuanetti and Dr. Peter B. Corr who generously provided canine epicardial border zone tissue. We thank Dr. Robert H. Hoyt for helpful scientific discussions, William Kraft and Timothy Tolley for technical assistance, Louise Schoelch and Margaret McHugh for photographic assistance, Dr. Kenneth Schechtman for statistical assistance, and Susan Johnson for preparation of the typescript.

This work was supported by National Heart, Lung and Blood Institute grants HL-36773 and HL-17646, SCOR in Ischemic Heart Disease. Dr. Saffitz is an Established Investigator of the American Heart Association.

\section{References}

1. Fozzard, H. A., and M. F. Arnsdorf. 1986. Cardiac electrophysiology. In The Heart and Cardiovascular System. H. A. Fozzard, E. Habert, R. B. Jennings, A. M. Katz, and H. F. Morgan, editors. Raven Press, New York. 1-25.

2. Spear, J. F., E. L. Michelson, and E. N. Moore. 1983. Reduced space constant in slowly conducting regions of chronically infarcted canine myocardium. Circ. Res. 53:176-185.

3. Spach, M. S., P. C. Dolber, and J. F. Heidlage. 1988. Influence of the passive anisotropic properties on directional differences in propagation following modification of the sodium conductance in human atrial muscle. A model of reentry based on anisotropic discontinuous propagation. Circ. Res. 62:811-832.

4. Spach, M. S., W. T. Miller, III, D. B. Geselowitz, R. C. Barr, J. M. Kootsey, and E. A. Johnson. 1981. The discontinuous nature of propagation in normal canine cardiac muscle. Evidence for recurrent discontinuities of intracellular resistance that affect the membrane currents. Circ. Res. 48:39-54.

5. Dillon, S. M., M. A. Allessie, P. C. Ursell, and A. L. Wit. 1988. Influences of anisotropic tissue structure and reentrant circuits in the epicardial border zone of subacute canine infarcts. Circ. Res. 63:182-206.
6. Spear, J. F., E. L. Michelson, and E. N. Moore. 1983. Cellular electrophysiologic characteristics of chronically infarcted myocardium in dogs susceptible to sustained ventricular tachyarrhythmias. J. Am. Coll. Cardiol. 1:1099-1110.

7. Spach, M. S., W. T. Miller, III, P. C. Dolber, J. M. Kootsey, J. R. Sommer, and C. E. Mosher, Jr. 1982. The functional role of structural complexities in the propagation of depolarization in the atrium of the dog. Cardiac conduction disturbances due to discontinuities of effective axial resistivity. Circ. Res. 50:175191.

8. Ursell, P. C., P. I. Gardner, A. Albala, J. J. Fenoglio, Jr., and A. L. Wit 1985. Structural and electrophysiological changes in the epicardial border zone of canine myocardial infarcts during infarct healing. Circ. Res. 56:436-451.

9. Friedman, P. L., J. J. Fenoglio, and A. L. Wit. 1975. Time course for reversal of electrophysiological and ultrastructural abnormalities in subendocardial Purkinje fibers surviving extensive myocardial infarction in dogs. Circ. Res. 36:127-144.

10. Richards, D. A., G. J. Blake, J. F. Spear, and E. N. Moore. 1984. Electrophysiologic substrate for ventricular tachycardia: correlation of properties in vivo and in vitro. Circulation. 69:369-381.

11. Gardner, P. I., P. C. Ursell, J. J. Fenoglio, and A. L. Wit. 1985. Electrophysiologic and anatomic basis for fractionated electrograms recorded from healed myocardial infarcts. Circulation. 72:596-611.

12. Surawicz, B. 1989. Ventricular arrhythmias: why is it so difficult to find a pharmacologic cure. J. Am. Coll. Cardiol. 14:1401-1416.

13. Wit, A. L., M. A. Allessie, F. I. M. Bonke, W. Lammers, J. Smeets, and J. J. Fenoglio. 1982. Electrophysiologic mapping to determine the mechanism of experimental ventricular tachycardia initiated by premature impulses. Experimental approach and initial results demonstrating reentrant excitation. Am. J. Cardiol. 49:166-185.

14. Wit, A. L. 1989. Anisotropic reentry: A model of arrhythmias that may necessitate a new approach to antiarrhythmic drug development. In Lethal arrhythmias resulting from myocardial ischemia and infarction. M. R. Rosen and Y. Palti, editors. Kluwer Academic Publishers, Boston. 199-213.

15. Spach, M. S., and P. C. Dolber. 1986. Relating extracellular potentials and their derivatives to anisotropic propagation at a microscopic level in human cardiac muscle-evidence for electrical uncoupling of side-to-side fiber connections with increasing age. Circ. Res. 58:356-371.

16. Hoyt, R. H., M. L. Cohen, and J. E. Saffitz. 1989. Distribution and threedimensional structure of intercellular junctions in canine myocardium. Circ. Res. 64:563-574.

17. Luke, R. A., E. C. Beyer, R. H. Hoyt, and J. E. Saffitz. 1989. Quantitative analysis of intercellular connections by immunohistochemistry of the cardiac gap junction protein connexin43. Circ. Res. 65:1450-1457.

18. Zuanetti, G., R. H. Hoyt, and P. B. Corr. 1990. $\beta$-Adrenergic-mediated influences on microscopic conduction in epicardial regions overlying infarcted myocardium. Circ. Res. 67:284-302.

19. Weibel, E. R. 1969. Stereological principles for morphometry in electron microscopic cytology. Int. Rev. Cytol. 26:235-299.

20. SAS Institute Inc. 1988. SAS/STAT TM User's Guide. Release 6.03 edition. SAS Institute Inc., Cary, NC.

21. Gerdes, A. M., and F. H. Kasten. 1980. Morphometric study of endomyocardium and epimyocardium of the left ventricle in adult dogs. Am. J. Anat. 159:389-394.

22. Phillips, S. J., E. A. Pappas, M. Paulosky, and D. Meir-Levi. 1981. Cellular connectivity between adjacent mammalian ventricular heart cells. Anat. Rec. 199:202A. (Abstr.)

23. DeBakker, J. M. T., F. J. L. van Capelle, M. J. Janse, A. A. M. Wilde, R. Coronell, A. E. Becker, K. P. Dingemans, N. M. van Hemel, and R. N. W. Hauer. 1988. Reentry as a cause of ventricular tachycardia in patients with chronic ischemic heart disease: electrophysiologic and anatomic correlation. Circulation. 77:589-606.

24. Cardinal, R., M. Vermeulsen, M. Shenasa, F. Roberge, P. Page, F. Helie and P. Savard. 1988. Anisotropic conduction and functional dissociation of ischemic tissue during reentrant ventricular tachycardia in canine myocardial infarction. Circulation. 77:1162-1176.

25. Kramer, J. B., J. E. Saffitz, F. X. Witkowski, and P. B. Corr. 1985. Intramural reentry as a mechanism of ventricular tachycardia during evolving canine myocardial infarction. Circ. Res. 56:736-754.

26. Wit, A. L., and M. E. Josephson. 1985. Fractionated electrograms and continuous electrical activity: fact or artifact. In Cardiac Electrophysiology and Arrhythmias. D. P. Zipes, and J. Jalife, editors. Grune and Stratton, New York. 343-351.

27. Waxman, H. L., and R. J. Sung. 1980. Significance of fragmented ventricular electrograms observed using intracardiac recording techniques in man. Circulation. 62:1349-1356.

28. Spach, M. S., R. C. Barr, E. A. Johnson, and J. M. Kootsey. 1973. Cardiac extracellular potentials. Analysis of complex waveforms about the Purkinje networks in dogs. Circ. Res. 33:465-473. 